\title{
Predicting response of severe aplastic anemia to immunosuppression combined with eltrombopag
}

Yoshitaka Zaimoku, ${ }^{1}$ Bhavisha A. Patel, ${ }^{1}$ Ruba Shalhoub, ${ }^{2}$ Emma M. Groarke, ${ }^{1}$ Xingmin Feng, ${ }^{1}$ Colin $\mathrm{O}$. Wu ${ }^{2}$ and Neal S. Young ${ }^{1}$

${ }^{1}$ Hematology Branch, National Heart, Lung, and Blood Institute, National Institutes of Health and ${ }^{2}$ Office of Biostatistics Research, National Heart, Lung, and Blood Institute,

Haematologica 2022

Volume 107(1):126-133

National Institutes of Health, Bethesda, MD, USA

\section{ABSTRACT}

P retreatment blood counts, particularly an absolute reticulocyte count $\geq 25 \times 10^{9} / \mathrm{L}$, correlate with response to immunosuppressive therapy in severe aplastic anemia. In recent trials, eltrombopag combined with standard immunosuppressive therapy yielded superior responses than those to immunosuppressive therapy alone. Our single institution retrospective study aimed to elucidate whether historical predictors of response to immunosuppressive therapy alone were also associated with response to immunosuppressive therapy plus eltrombopag. We sought correlations of blood counts, thrombopoietin levels and the presence of paroxysmal nocturnal hemoglobinuria clones with both overall and complete responses in 416 patients with severe aplastic anemia, aged 2-82 years (median, 30 years), initially treated with immunosuppressive therapy plus eltrombopag between 2012 and $2019(n=176)$ or with immunosuppressive therapy alone between 1999 and 2010 $(n=240)$. Compared to non-responders, patients in the group of overall responders to immunosuppressive therapy plus eltrombopag had significantly higher pretreatment absolute reticulocyte counts, higher neutrophil counts and reduced thrombopoietin levels, as also observed for the group treated with immunosuppressive therapy alone. Addition of eltrombopag markedly improved the overall response in subjects with an absolute reticulocyte count between $10-30 \times 10^{\%} / \mathrm{L}$ from $60 \%$ (54 of 90 ) to $91 \%$ (62 of 68). Absolute lymphocyte count correlated with complete response in the groups treated with immunosuppressive therapy with or without eltrombopag, especially in adolescents aged $\geq 10$ years and adults, but the correlation was reversed in younger children. Platelet count and the presence of a paroxysmal nocturnal hemoglobinuria clone did not correlate with responses to immunosuppressive therapy. Blood counts remain the best predictors of response to nontransplant therapies in severe aplastic anemia. Addition of eltrombopag to immunosuppressive therapy shifted patients with a lower absolute reticulocyte count into a better prognostic category.

https://doi.org/10.3324/haematol.2021.278413

(C)2022 NIH

\section{Introduction}

Immune aplastic anemia (AA), characterized by T-cell-mediated destruction of hematopoietic stem and progenitor cells, is successfully treated with hematopoietic stem cell transplantation (HSCT) or immunosuppressive therapy (IST). ${ }^{1}$ HSCT is preferred for severe AA (SAA) in children and young adults aged $\leq 40$ years, if an HLA-matched sibling donor is available, as it is usually a curative option and has acceptable toxicities. Most adult patients undergo IST since transplantation-related mortality increases with age. ${ }^{2}$ IST may result in incomplete hematologic recovery, relapse, or clonal evolution to a myeloid malignancy. Standard IST has been established as horse antithymocyte globulin (hATG) plus cyclosporine, to which $60-70 \%$ of patients respond with clinically meaningful hematologic improvement. Experimental clinical protocols conducted at our institution using rabbit ATG, alemtuzumab, cyclophosphamide, or with the addition to standard treatment of 
mycophenolate mofetil and sirolimus, and by others with the addition of androgens, granulocyte - colony-stimulating factor and erythropoietin to standard IST have failed to demonstrate superiority to standard hATG and cyclosporine. ${ }^{3}$ However, eltrombopag, a synthetic thrombopoietin receptor agonist, when added to standard IST resulted in better outcomes compared to the standard regimen, with an overall response rate of $>80 \%$ and 2-year survival rate of $97 \%,{ }^{4}$ and this combination is now approved for first-line use in SAA in the USA. A large, prospective, randomized trial comparing standard IST to IST plus eltrombopag has broadly confirmed these results, ${ }^{5}$ and smaller case series in a variety of populations have also suggested improved outcomes with the addition of thrombopoietin mimetics. ${ }^{6-11}$

Response to conventional ATG-based IST without eltrombopag correlates with blood counts before therapy, including absolute reticulocyte count (ARC), ${ }^{12-18}$ absolute neutrophil count (ANC) $)^{12,16,17,19,20}$ and absolute lymphocyte count (ALC) ${ }^{12,21,22}$ The presence of somatic mutations, ${ }^{23}$ having a paroxysmal nocturnal hemoglobinuria (PNH) clone, ${ }^{13,15,20,2,24,25}$ elevated thrombopoietin levels, ${ }^{16}$ normal telomere length of lymphocytes, ${ }^{15,26}$ a subpopulation of regulatory $\mathrm{T}$ cells $s^{27}$ and $\mathrm{B}$ cells ${ }^{28}$ have all been reported as prognostic markers of response. Studies typically have emphasized overall response rather than complete response, but a positive feature of IST combined with eltrombopag is the high complete response rate. ${ }^{4}$ Previously, the robustness of response has been correlated with better overall survival. ${ }^{29}$

The aim of this study was to elucidate whether IST response predictors before the introduction of eltrombopag remain associated with predictors of treatment response with IST plus eltrombopag and to identify subgroups of patients with SAA that might particularly benefit from IST plus eltrombopag. We sought correlations of age, initial blood counts, thrombopoietin levels, and the presence of PNH clones with both the overall and complete responses in a large dataset including groups of patients with SAA treated on consecutive clinical protocols with IST alone and IST plus eltrombopag at the National Heart, Lung, and Blood Institute (NHLBI), National Institutes of Health (NIH).

\section{Methods}

\section{Patients and treatment}

Patients with SAA, aged $\geq 2$ years, treated with hATG-based regimens at the NIH Clinical Center, Bethesda, between May 1999 and August 2019, were included in the analysis. Studies before 1999 were excluded as information on complete response was not available. The patients were treated on any of four clinical studies approved by the Institutional Review Board of the NHLBI (NCT00001964, NCT00061360, NCT00260689, NCT01623167) and were done under the principles of the Declaration of Helsinki. Informed consent was obtained on the respective protocols.

Treatment regimens have been published:4,30-32 hATG (ATGAM, Pfizer) and cyclosporine were administered to all the patients; an investigational agent, including mycophenolate mofetil, sirolimus or eltrombopag, was added to IST in some protocols (Online Supplementary Methods and Online Supplementary Figure S1). The IST plus eltrombopag group referred to four cohorts administered eltrombopag in addition to
hATG and cyclosporine; the IST alone group included other hATG-based IST.

\section{Definitions}

All definitions have been consistent across all our protocols. SAA was diagnosed when bone marrow cellularity was $<30 \%$ and at least two of the following three criteria were met: ANC $<0.5 \times 10^{9} / \mathrm{L}$, ARC $<60 \times 10^{9} / \mathrm{L}$, and platelet count $<20 \times 10^{9} / \mathrm{L}$. Hematologic responses were evaluated 6 months after administration of ATG. Overall response was defined as no longer meeting the criteria for SAA in the absence of recent transfusions and granulocyte colony-stimulating factor administration; complete response required all ANC $\geq 1.0 \times 10^{9} / \mathrm{L}$, hemoglobin level $\geq 10$ $\mathrm{g} / \mathrm{dL}$ and platelet count $\geq 100 \times 10^{9} / \mathrm{L}$; and partial response was defined as an overall response not meeting the criteria for complete response. For the purpose of statistical analysis, patients who did not complete 6 months of initial IST due to death, HSCT or who underwent a second course of IST were counted as having had no response, consistent with our previous study. ${ }^{12}$ Blood values assessed as potential response predictors were ARC, ANC, ALC, platelet count, presence of a PNH clone and plasma thrombopoietin levels. The lowest values during the 4 weeks preceding IST were used as pretreatment blood counts, as previously described. ${ }^{12}$ Plasma thrombopoietin levels were measured by magnetic Luminex assay with a standard proto$\mathrm{col}^{33} \mathrm{PNH}$ clones were defined as the presence of $1 \%$ or more clonal populations deficient in glycosylphosphatidylinositol anchored proteins on the surface of neutrophils or red blood cells; information on PNH clones was available only for neutrophils in the IST plus eltrombopag study.

\section{Statistics}

Statistical analyses were performed using an EZR software package (version 1.41), graphical user interphase of $\mathrm{R}$ (version 3.6.1). ${ }^{34}$ The Fisher exact test and Mann-Whitney $U$ test were used to compare categorical and numerical variables, respectively. Correlations between baseline numerical variables were tested with the Spearman rank correlation test. In the logistic regression model, continuous variables were transformed to the natural $\log (X+1)$, where $X$ represents the variable, consistent with the previous study. ${ }^{12}$ All variables with $P<0.2$ in the univariate analysis were included in the multivariate analysis and a backward stepwise selection procedure was performed to create the final model.

\section{Results}

\section{Patients}

A total of 417 patients with SAA, aged 2 to 82 years, were initially treated with standard hATG and cyclosporine, with or without an investigational agent, between May 1999 and August 2019, on any of four prospective studies (Online Supplementary Figure S1): (i) a single arm study of standard IST plus mycophenolate mofetil performed from May 1999 to June 2003 ( $n=103) ;^{30}$ (ii) a direct comparison of standard IST $(n=42)$ and IST plus sirolimus ( $n=35)$, from June 2003 to November 2005; ${ }^{31}$ (iii) a direct comparison of standard IST $(n=60)$ and rabbit ATG-based IST ( $n=60$; not included), from December 2005 to July $2010 ;^{32}$ and (iv) a single-arm study of IST plus eltrombopag ( $n=177)$, from July 2012 to August 2019, including cohort $1(n=30)$, cohort $2(n=31)$, cohort $3(n=31)$ and an extension cohort after the publication $(n=85) .{ }^{4}$ One patient who received IST plus eltrombopag in the exten- 
sion cohort did not reach the 3-month evaluation after a hematologic response and was excluded from the statistical analysis.

Data on PNH clones were not available for 23 subjects: 19 in the IST plus eltrombopag group were not analyzable as their ANC were too low, at a median (IOR) of 0 (0$0.01) \times 10^{9} / \mathrm{L}$; for the other four treated with IST plus mycophenolate mofetil, PNH clone information was missing. Thrombopoietin levels were quantified in 140 of the 176 patients treated with IST plus eltrombopag; we used serum samples instead of plasma in nine patients, as levels of thrombopoietin in serum and plasma obtained at the same time were equivalent (in 15 patients with $\mathrm{AA}$; correlation coefficient of 0.95 ; Wilcoxon signed rank test, $P=0.26$ ) (Online Supplementary Figure S2).

The clinical characteristics of the patients are summarized in Table 1. The 176 patients treated with IST plus eltrombopag showed significantly higher ARC and lower platelet counts compared to the 240 patients treated with IST alone; age, sex, ANC, ALC and the prevalence of PNH clones were well balanced between the two groups. The IST plus eltrombopag group had significantly higher overall and complete response rates than did the group treated with IST alone: overall response of $82 \%$ (144 of 176) versus $62 \%$ (149 of $240, P<0.0001$ ) and complete response of $39 \%$ (69 of 176 ) versus $13 \%$ (32 of $240, P<0.0001$ ), as previously published. ${ }^{4}$ There were no significant differences in the response rates across the cohorts in the IST plus eltrombopag group and across the non-eltrombopag regimens, except for the absence of complete response in 35 patients treated with standard IST plus sirolimus (Online Supplementary Figure S1).

\section{Correlates of overall response}

Patients who responded to IST plus eltrombopag, as compared to those who did not, showed significantly higher ARC (median [IOR], $21[10-36] \times 10^{\%} / \mathrm{L}$ vs. 9 [7$\left.22] \times 10^{9} / \mathrm{L}, \quad P=0.00090\right)$, higher ANC $(0.33 \quad[0.14-$ $0.53] \times 10^{9} / \mathrm{L}$ vs. $\left.0.06[0.01-0.25] \times 10^{9} / \mathrm{L}, P=0.00027\right)$ and a tendency to higher ALC $\left(1.29[0.99-1.65] \times 10^{\circ} / \mathrm{L}\right.$ vs. 1.00 $\left.[0.59-1.46] \times 10^{9} / \mathrm{L}, P=0.079\right)$ before therapy, similar to the group treated with IST alone (Figure 1; Table 2). Plasma thrombopoietin levels were significantly elevated in nonresponders to IST plus eltrombopag $(3,000$ [2,610-3,430] $\mathrm{ng} / \mathrm{mL}$ vs. $2,540[2,200-2,950] \mathrm{ng} / \mathrm{mL}, P=0.0037)$, as reported by others for IST alone. ${ }^{16}$ Age, sex, platelet counts or the presence of $\mathrm{PNH}$ clones did not correlate significantly with overall response to IST with or without eltrombopag. Multivariate logistic regression analysis revealed that ARC and ALC were independent predictors of overall response among all the 416 patients, consistent with the findings of a previous study:; ARC and thrombopoietin were retained in the final model of 176 patients in the IST

Table 1. Patients' characteristics at baseline.

\begin{tabular}{|c|c|c|c|}
\hline & IST+EPAG $(n=176)$ & IST alone ( $n=240)$ & $P$ \\
\hline $\begin{array}{l}\text { Age, years } \\
\text { Median (IQR) } \\
\text { Range }\end{array}$ & $\begin{array}{c}32(19-55) \\
3-82\end{array}$ & $\begin{array}{c}30(18-53) \\
2-82\end{array}$ & 0.47 \\
\hline $\begin{array}{l}\text { Sex } \\
\quad \text { Male } \\
\text { Female }\end{array}$ & $\begin{array}{l}87(49 \%) \\
89(51 \%)\end{array}$ & $\begin{array}{c}141(59 \%) \\
99(41 \%)\end{array}$ & 0.073 \\
\hline $\begin{array}{l}\text { Blood count, } \times 10^{9} / \mathrm{L} \\
\text { ARC } \\
\text { ANC } \\
\text { ALC } \\
\text { Platelets }\end{array}$ & $\begin{array}{c}18.8(8.7-34.4) \\
0.28(0.078-0.51) \\
1.26(0.93-1.61) \\
8.0(4.8-11.3)\end{array}$ & $\begin{array}{c}14.3(4.9-30.7) \\
0.30(0.11-0.50) \\
1.28(0.90-1.66) \\
9.0(6.0-13)\end{array}$ & $\begin{array}{c}0.0086 \\
0.36 \\
0.89 \\
0.031\end{array}$ \\
\hline TPO, $\mathrm{pg} / \mathrm{mL}^{*}$ & $2610(2220-3080)$ & NA & \\
\hline $\begin{array}{l}\text { PNH clone }^{\dagger} \\
\quad \geq 1 \% \\
\quad<1 \%\end{array}$ & $\begin{array}{l}60(38 \%) \\
97(62 \%)\end{array}$ & $\begin{array}{c}90(38 \%) \\
146(62 \%)\end{array}$ & 1 \\
\hline $\begin{array}{l}\text { Response at } 6 \text { months } \\
\text { Overall response } \\
\text { Complete response }\end{array}$ & $\begin{array}{l}144(82 \%) \\
69(39 \%)\end{array}$ & $\begin{array}{l}149(62 \%) \\
32(13 \%)\end{array}$ & $\begin{array}{l}<0.0001 \\
<0.0001\end{array}$ \\
\hline
\end{tabular}

Data are $\mathrm{n}(\%)$ or median (interquartile range) unless otherwise noted. IST: immunosuppressive therapy; EPAG: eltrombopag; IQR: interquartile range; ARC: absolute reticulocyte count;ANC: absolute neutrophil count;ALC: absolute lymphocyte count;TPO thrombopoietin; PNH: paroxysmal nocturnal hemoglobinuria; NA: not assessed. *TPO was studied in 140 patients treated with IST+EPAG. 'The PNH clone was not tested in 19 and four patients treated with IST plus EPAG and IST alone, respectively.
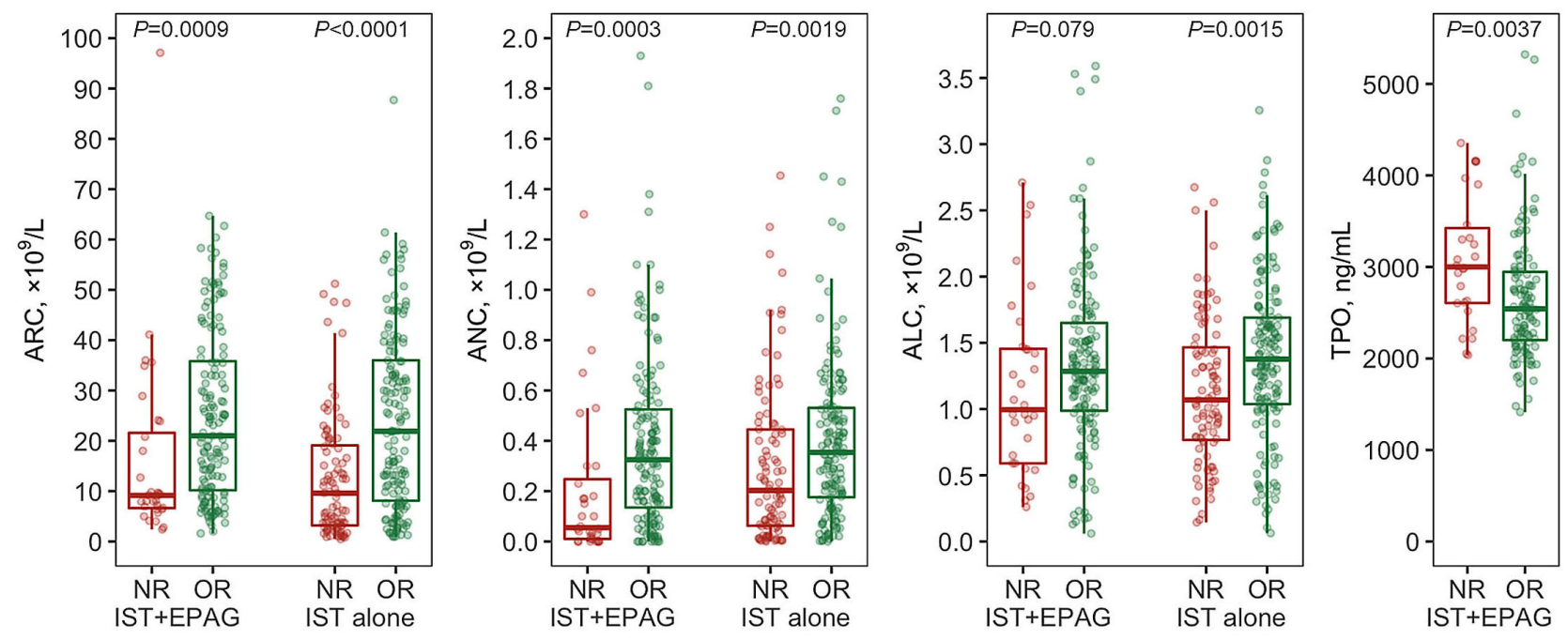

Figure 1. Pretreatment blood counts and overall response. Absolute reticulocyte count, absolute neutrophil count, absolute lymphocyte count and plasma thrombopoietin level at baseline were compared between patients who achieved a response and those who did not at 6 months according to whether they were treated with immunosuppressive therapy plus eltrombopag or immunosuppressive therapy alone. ARC: absolute reticulocyte count; ANC: absolute neutrophil count; ALC: absolute lymphocyte count; TPO: thrombopoietin; NR: no response; OR: overall response; IST: immunosuppressive therapy; EPAG: eltrombopag. 
plus eltrombopag group (Online Supplementary Table S1).

Although not statistically significant, the patients with PNH clones showed slightly higher overall response in both IST groups (odds ratio $1.32, P=0.24$ ) (Online Supplementary Table S1), consistent with previous studies, but the favorable tendency disappeared in the multivariate model (odds ratio $1.06, P=0.87$ ), as the presence of $\mathrm{PNH}$ clones correlated with higher ALC $(P=0.018)$ and higher ARC $(P=0.073)$. We also noted that the 19 patients who did not have enough ANC to assess PNH clones showed a significantly reduced overall response to IST plus eltrombopag at $53 \%$ (10 of $19 ; P=0.0019$ ), which was analogous to the response rate in 19 patients with ANC $<0.02 \times 10^{9} / \mathrm{L}$ at baseline.

The response curves revealed that the reduction of overall response to IST plus eltrombopag was observed only in patients with pre-treatment ARC lower than $10 \times 10^{9} / \mathrm{L}$ while overall response to IST alone decreased below an ARC of $30 \times 10^{9} / \mathrm{L}$ (Figure $2 \mathrm{~A}$ ). The overall response in subjects with ARC between $10-30 \times 10^{9} / \mathrm{L}$ was significantly improved in the group treated with IST plus eltrombopag, to the levels of those of patients with ARC $>30 \times 10^{9} / \mathrm{L}$ (from $60 \%$ to $91 \%, P<0.0001$ ) (Figure $2 \mathrm{~B}$ ). Addition of eltrombopag did not appear to significantly improve the overall response in subjects with ARC $<10 \times 10^{\circ} / \mathrm{L}$ ( $63 \%$ in the IST plus eltrombopag group and $46 \%$ in the IST alone group, $P=0.062$ ) nor in those with ARC $>30 \times 10^{9} / \mathrm{L}(90 \%$ vs. $89 \%, P=1)$, but was beneficial in terms of increasing complete response $(27 \%$ vs. $8 \%$ in the groups with $\mathrm{ARC}<10 \times 10^{\circ} / \mathrm{L}, P=0.0036 ; 42 \%$ vs. $21 \%$ in

Table 2. Pretreatment variables and responses to immunosuppressive therapy.

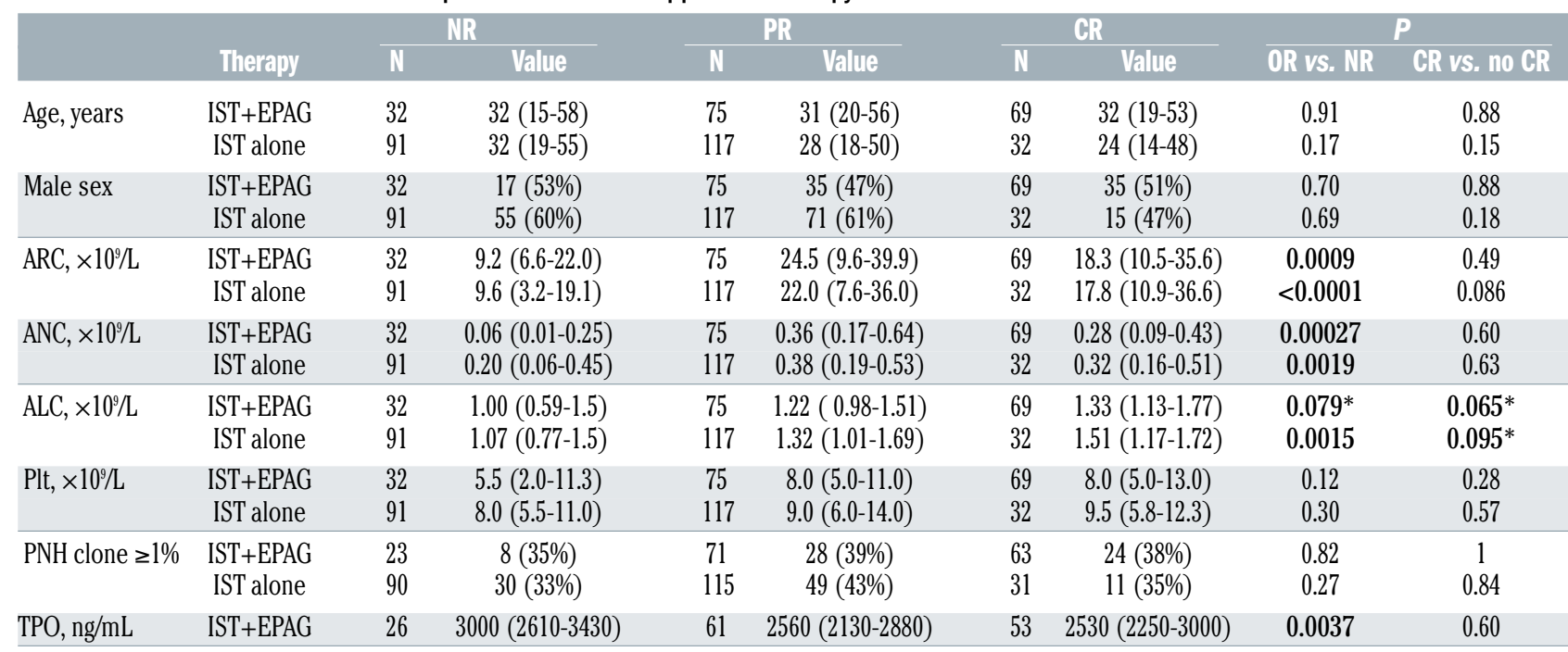

Data are $\mathrm{n}(\%)$ or median (interquartile range) unless otherwise noted. NR: no response; PR: partial response; CR: complete response; IST: immunosuppressive therapy; EPAG, eltrombopag;ARC: absolute reticulocyte count;ANC: absolute neutrophil count;ALC: absolute lymphocyte count; Plt:platelet count; PNH:paroxysmal nocturnal hemoglobinuria; TPO: thrombopoietin. ${ }^{*} P<0.05$ in patients aged $\geq 10$ years.

A

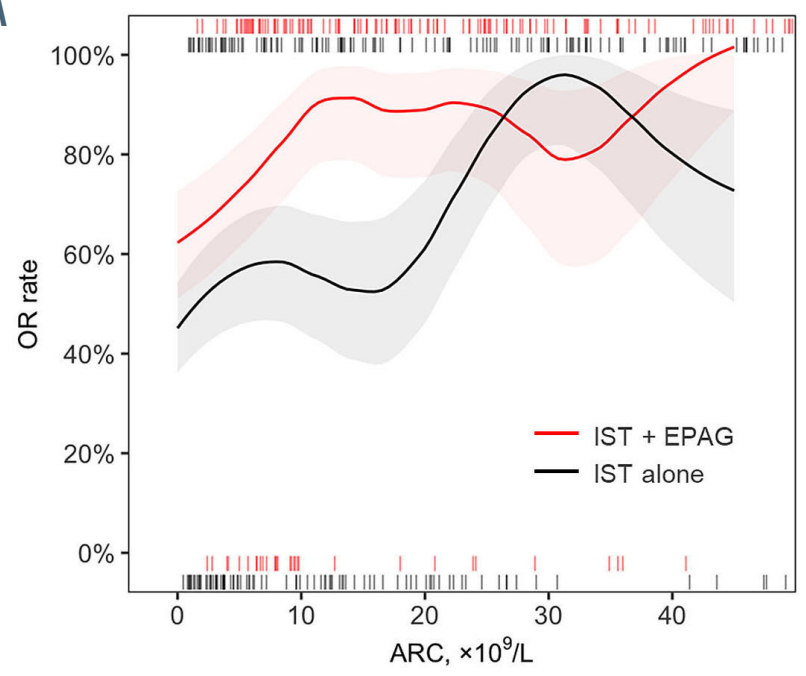

B

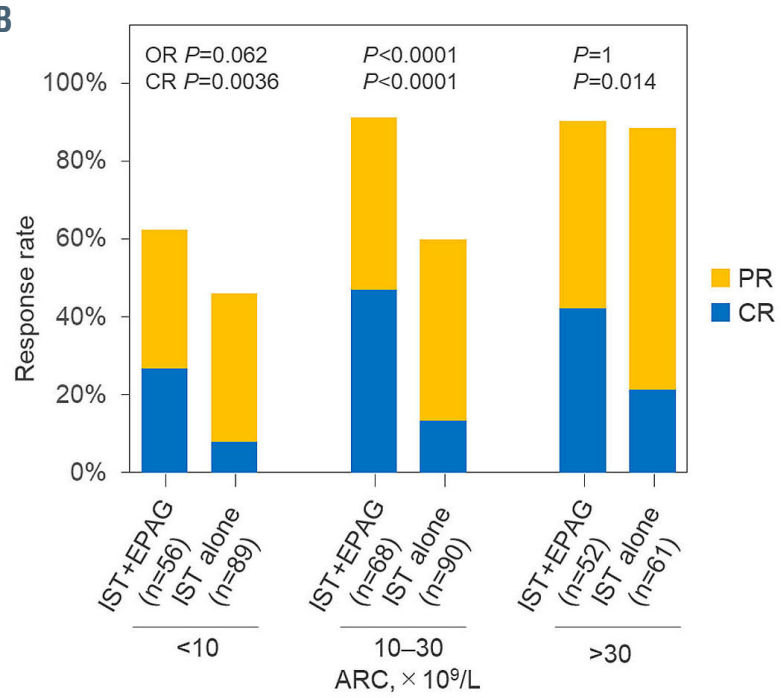

Figure 2. Pretreatment absolute reticulocyte count and response rates to immunosuppressive therapy. (A) The overall response rates and their $90 \%$ confidence interval of individuals with an initial absolute reticulocyte count between $X$ and $(X+10) \times 10 \%$ in the group treated with immunosuppressive therapy (IST) plus eltrombopag (EPAG) and in the group treated with IST alone. (B) The overall response rates and the complete response rates were compared between the IST plus EPAG group and IST alone group according to initial absolute reticulocyte count. Overall response includes complete responses and partial responses. OR: overall response; ARC: absolute reticulocyte count; PR: partial response; CR: complete response. 
the group with $\left.\mathrm{ARC}>30 \times 10^{9} / \mathrm{L}, P=0.024\right)$ (Figure $2 \mathrm{~B}$ ). There was no such clear difference of response curve between the IST groups according to ANC or ALC (Online Supplementary Figure S3).

\section{Prediction of complete response}

Pretreatment ALC was especially elevated in patients who achieved a complete response in both treatment groups, IST plus eltrombopag and IST alone ( $P$-values, 0.065 and 0.095 , compared to no complete response) (Figure 3; Table 2), which was statistically significant in all patients combined $(P=0.020)$. Other variables, including the strong predictors of overall response, were unexpectedly not discriminatory for complete response.

A large retrospective study of 312 pediatric AA patients found significantly reduced pretreatment ALC in IST responders compared to non-responders (median of $1.6 \times 10^{9} / \mathrm{L}$ vs. $2.0 \times 10^{9} / \mathrm{L}$, respectively; $\left.P=0.006\right) ;{ }^{21}$ and other studies in children also showed similar trends, while the correlation of higher ALC with better outcomes was mostly described in adult patients (Online Supplementary Table S2). We therefore hypothesized that the impact of ALC on IST responsiveness differed by age. We did observe a significant inverse correlation of ALC with complete response in 34 younger children aged $<10$ years for the two IST groups; six complete responders compared to the other 28 patients had significantly reduced ALC at a median (IOR) of $0.41(0.31-1.25) \times 10^{9} / \mathrm{L}$ versus 1.50 (1.07$2.13) \times 10^{9} / \mathrm{L}(P=0.037)$ (Online Supplementary Figure S4A). ALC retained a significant positive correlation with complete response in adolescents aged $10-19$ years $(P=0.012)$ and in adults aged 20 years or older $(P=0.029)$. Patient age of 10 years was the best cutoff to discriminate the inverse relationship of ALC with complete response in younger and older individuals (Online Supplementary Figure S4B-D). Therefore, exclusion of the young children aged $<10$ years from the statistical analyses led to a significant correlation of ALC with complete response $(P=0.010$ in the IST plus eltrombopag group and $P=0.012$ in the group treated with
IST alone) as well as with overall response ( $P=0.016$ in the IST plus eltrombopag group and $P=0.00011$ in the IST alone group). Complete response tended to associate with higher ALC in all the age groups over 10 years old, but the association was especially obvious in adolescents and young adults, aged 10-40 years (Online Supplementary Figure $S 4 A$ ). The complete response rate to IST plus eltrombopag in the adolescents and young adults reached $60 \%$ (28 of 47 ) if the ALC was more than $1.3 \times 10^{\circ} / \mathrm{L}$ before therapy, which was significantly higher than the $32 \%$ (15 of $47, P=0.013)$ in the other half of patients with lower ALC; this cut-off of ALC could also stratify complete response probability in the group treated with IST alone (21\% [14 of 68] vs. $3 \%$ [2 of 66]; $P=0.0024)$.

We did not observe such an age-dependent inverse correlation in other predictors of overall response (Online Supplementary Figure S5).

\section{Rabbit antithymocyte globulin-based immunosuppres- sive therapy}

Because the correlation of ALC with complete response has not been reported, we additionally assessed this relationship in 54 patients, aged 10 years or older, treated with rabbit ATG and cyclosporine (NCT00260689). ${ }^{32}$ Four of the 54 patients achieved a complete response; they had significantly higher ALC compared to the remaining 50 patients at a median (IOR) of $1.97(1.74-2.41) \times 10^{9} / \mathrm{L}$ versus $1.14(0.81-1.36) \times 10^{9} / \mathrm{L}(P=0.0045)$ (Online Supplementary Figure S6). None of six children aged $<10$ years treated with rabbit ATG-based IST achieved a complete response. ARC and ANC did not correlate with complete response, but as expected they did with overall response, similar to hATG-based IST.

\section{Discussion}

Eltrombopag combined with standard IST is now the best nontransplant therapy for SAA, but little has been
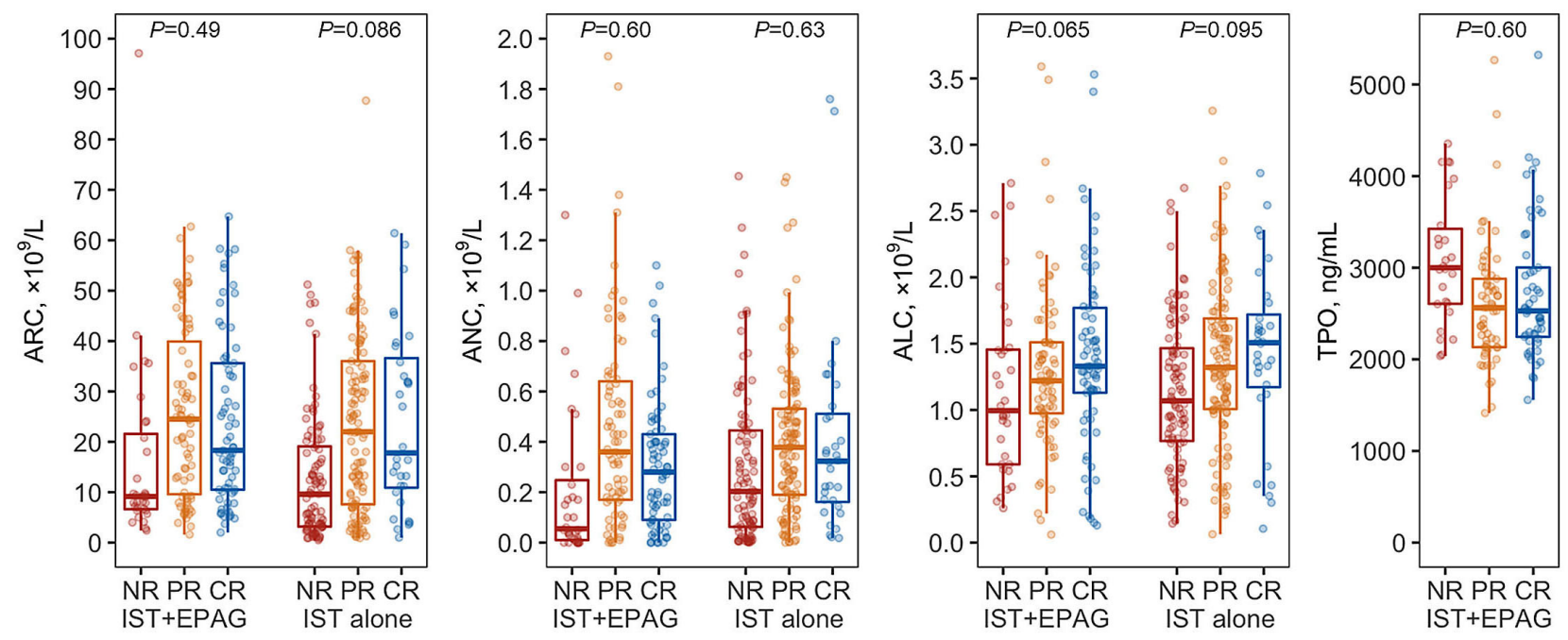

Figure 3. Blood counts and complete response. Pretreatment absolute reticulocyte count, absolute neutrophil count, absolute lymphocyte count and plasma thrombopoietin level of patients in the groups treated with immunosuppressive therapy (IST) plus eltrombopag (EPAG) and IST alone are shown according to responses (complete, partial and none) at 6 months. Initial absolute lymphocyte count tended to be elevated in patients who achieved complete response after IST both with and without EPAG, but absolute reticulocyte count, absolute neutrophil count and thrombopoietin, could not discriminate who would go on to have a complete response. ARC: absolute reticulocyte count; ANC: absolute neutrophil count; ALC: absolute lymphocyte count; TPO: plasma thrombopoietin; NR: no response; PR: partial response; CR: complete response. 
reported concerning response predictors, partly because of the very limited number of non-responders after such highly effective therapy. Our large retrospective study in 177 SAA patients treated with IST plus eltrombopag, incorporating 85 new subjects after the initial publication, ${ }^{4}$ has now revealed that pretreatment blood counts remain strong predictors of overall response to IST plus eltrombopag, similar to their predictive value in 240 patients treated with IST alone. We also noted the relationship of ALC with complete response for the first time in both groups of patients, i.e., those treated with IST plus eltrombopag and those treated with IST alone.

The overall response rate to IST plus eltrombopag exceeded $90 \%$ in patients who had an ARC of at least $10 \times 10 \% / \mathrm{L}$, and the complete response rate reached $60 \%$ in adolescents and young adults who had an ALC of more than $1.3 \times 10^{9} / \mathrm{L}$ prior to treatment. The addition of eltrombopag to IST markedly improved responses in patients who had an $\mathrm{ARC}$ in the $10-30 \times 10^{9} / \mathrm{L}$ range. In contrast, the addition of eltrombopag to IST did not significantly increase overall response in patients who had an ARC lower than $10 \times 10^{9} / \mathrm{L}$ or higher than $30 \times 10^{9} / \mathrm{L}$, but was still beneficial as it induced significantly more complete responses compared to IST only.

Multiple studies have attempted to predict response to IST in patients with SAA because HSCT is available as a competing treatment option. In practice, only the patient's age, in addition to the availability of an appropriate donor, is generally considered to decide initial therapy. ${ }^{2}$ This algorithm is based on outcomes of patients treated with IST alone, ${ }^{35,36}$ and may need to be revised in the eltrombopag era. In the short-term, patients with residual hematopoiesis might benefit more from IST plus eltrombopag than from HSCT, but long-term follow-up especially of relapse and clonal evolution with this new combination is necessary and is currently underway. The impact of pre-transplant blood counts on the outcomes after HSCT needs to be studied as well in AA.

The correlations of initial ARC and ANC with overall resposne were not surprising, as residual hematopoietic stem and progenitor cells should have an impact on hematopoietic recovery after immunosuppression, and they themselves are criteria for response. Similarly, the impact of thrombopoietin levels would be attributable to their inverse correlation with platelet production, ${ }^{37,38}$ another overall response criterion, although pretreatment platelet counts, affected by platelet transfusion, were not predictive of responses to IST.

Complete response was unexpectedly not discriminated by the strong overall response predictors, including ARC, ANC and thrombopoietin levels; these may not represent the quantity or quality of hematopoietic stem cells needed for complete hematologic recovery, as blood counts of patients with AA are often supported by aberrant hematopoiesis from $\mathrm{PNH}$ clones, ${ }^{24,39}$ HLA class I allele lacking clones, ${ }^{40-42}$ or clones associated with agerelated somatic mutations. ${ }^{23}$ These are usually detected in myeloid and erythroid cells but are less frequent or absent in lymphocytes. ${ }^{43-45}$

We observed an association of ALC with complete response and further validated the correlation in rabbit ATG-based IST. ALC may be more representative of levels of residual hematopoietic stem cells than other blood counts. On the other hand, recent studies in HSCT indi- cated that ATG dose adjustment based on ALC, rather than body weight, is beneficial to achieve an optimal level of immunosuppression because circulating lymphocytes bind and clear ATG; $;^{46-49}$ a low pretreatment ALC leads to reduced clearance of ATG and prolonged immunesuppression, which is likely unfavorable for hematologic recovery, as observed in patients treated with rabbit ATG-based IST. ${ }^{32}$ The impact of ALC was age-dependent, as complete response was associated with lower ALC in young children aged $<10$ years, but with higher ALC in adolescents and adult patients. Our literature review supported the differential impact of pretreatment ALC in children and in adults. The difference by age is probably associated with higher normal ALC and higher lymphoproliferative capacity in young children than in adolescents and adults; ${ }^{50,51}$ our body weight-based ATG dosage might be insufficient to suppress proliferative lymphocytes of young children unless ALC is markedly reduced before treatment.

A tendency to a slightly higher overall response in patients with $\mathrm{PNH}$ clones was attributed to the correlations of the presence of PNH clones with higher ALC and ARC, which may represent escape hematopoiesis by $\mathrm{PNH}$-phenotype hematopoietic stem cells and underestimation of PNH clones in very severe disease in which neutrophils are absent and red blood cells are replaced by transfusion. A lower threshold allows detection of patients having small PNH clones but also creates more difficulties in very severe disease as many more cells are needed to identify the small populations. Care must be taken to interpret biomarker studies on prediction of response to IST, as they are also potentially influenced by the blood counts. For example, clonal hematopoiesis that preferentially involves myeloid progenitors will be underestimated in patients with low ANC; thrombopoietin levels show an inverse correlation with platelet production; $;^{37,38}$ and lymphocyte subsets also corelate with myelopoiesis. $^{28}$

In summary, residual hematopoiesis, as indicated by baseline ARC, ANC and thrombopoietin, is predictive of initial overall response to eltrombopag plus IST, but does not correlate with complete response. We did, however, observe an age-dependent association between complete response and ALC. These findings should be helpful for future protocols and in decision-making regarding treatments for individual patients.

\section{Disclosures \\ No conflicts of interest to disclose.}

\section{Contributions}

$Y Z, P A B$ and NSY designed the study and wrote the first draft of the manuscript. PAB, EMG and NSY participated in the patients' care. $Y Z, R S$ and COW analyzed the data. YZ and XF quantified thrombopoietin levels. All authors critically reviewed the draft and approved the final version for publication.

\section{Funding}

This investigation was supported by the Intramural Research Program of the NIH and the NHLBI. NCT01623167 is funded by Novartis and previously by GSK through a cooperative research and development agreement (CRADA).

\section{Data-sharing statement}

Individual patients' information will not be disclosed. 


\section{References}

1. Young NS. Aplastic anemia. N Engl J Med. 2018;379(17):1643-1656

2. Bacigalupo A. How I treat acquired aplastic anemia. Blood. 2017;129(11):1428-1436.

3. Scheinberg P, Young NS. How I treat acquired aplastic anemia. Blood. 2012;120 (6):1185-1196.

4. Townsley DM, Scheinberg P, Winkler T, et al. Eltrombopag added to standard immunosuppression for aplastic anemia. N Engl J Med. 2017;376(16):1540-1550.

5. Peffault de Latour R, Marsh J, Iacobelli S, et al. Results of the EBMT SAAWP phase III prospective randomized multicenter RACE study of horse ATG and ciclosporin with or without eltrombopag in naïve SAA patients [abstract]. In: 46th Annual Meeting of the EBMT. 2020 Aug 31-Sep 1; Virtual: 2020. Abstract O018.

6. Olnes MJ, Scheinberg P, Calvo KR, et al. Eltrombopag and improved hematopoiesis in refractory aplastic anemia. N Engl J Med. 2012;367(1):11-19.

7. Desmond R, Townsley DM, Dumitriu B, et al. Eltrombopag restores trilineage hematopoiesis in refractory severe aplastic anemia that can be sustained on discontinuation of drug. Blood. 2014;123(12):18181825.

8. Assi R, Garcia-Manero G, Ravandi F, et al. Addition of eltrombopag to immunosuppressive therapy in patients with newly diagnosed aplastic anemia. Cancer. 2018:124(21):4192-4201.

9. Lengline E, Drenou B, Peterlin $\mathrm{P}$, et al. Nationwide survey on the use of eltrombopag in patients with severe aplastic anemia: a report on behalf of the French Reference Center for Aplastic Anemia. Haematologica. 2018;103(2):212-220.

10. Lee JW, Lee SE, Jung CW, et al. Romiplostim in patients with refractory aplastic anaemia previously treated with immunosuppressive therapy: a dose-finding and long-term treatment phase 2 trial. Lancet Haematol. 2019;6(11):e562-e572.

11. Ecsedi M, Lengline É, Knol-Bout C, et al. Use of eltrombopag in aplastic anemia in Europe. Ann Hematol. 2019;98(6):1341-1350.

12. Scheinberg P, Wu CO, Nunez O, Young NS. Predicting response to immunosuppressive therapy and survival in severe aplastic anaemia. Br J Haematol. 2009;144(2):206216.

13. Afable MG 2nd, Shaik M, Sugimoto Y, et al. Efficacy of rabbit anti-thymocyte globulin in severe aplastic anemia. Haematologica. 2011:96(9):1269-1275.

14. Shin SH, Yoon JH, Yahng SA, et al. The efficacy of rabbit antithymocyte globulin with cyclosporine in comparison to horse antithymocyte globulin as a first-line treatment in adult patients with severe aplastic anemia: a single-center retrospective study. Ann Hematol. 2013;92(6):817-824.

15. Narita A, Muramatsu H, Sekiya Y, et al. Paroxysmal nocturnal hemoglobinuria and telomere length predicts response to immunosuppressive therapy in pediatric aplastic anemia. Haematologica. 2015;100 (12):1546-1552

16. Elmahdi S, Muramatsu H, Narita A, et al. Markedly high plasma thrombopoietin (TPO) level is a predictor of poor response to immunosuppressive therapy in children with acquired severe aplastic anemia. Pediatr Blood Cancer. 2016;63(4):659-664.

17. Vaht K, Goransson M, Carlson K, et al. Low response rate to ATG-based immuno- suppressive therapy in very severe aplastic anaemia - a Swedish nationwide cohort study. Eur J Haematol. 2018;100(6):613620.

18. Cabannes-Hamy A, Boissel N, Peffault De Latour $\mathrm{R}$, et al. The effect of age in patients with acquired aplastic anaemia treated with immunosuppressive therapy: comparison of Adolescents and Young Adults with children and older adults. $\mathrm{Br} \mathrm{J}$ Haematol. 2018;183(5):766-774

19. Chang $\mathrm{MH}$, Kim $\mathrm{KH}$, Kim HS, et al. Predictors of response to immunosuppressive therapy with antithymocyte globulin and cyclosporine and prognostic factors for survival in patients with severe aplastic anemia. Eur J Haematol. 2010;84(2):154-159.

20. Zhao X, Zhang L, Jing L, et al. The role of paroxysmal nocturnal hemoglobinuria clones in response to immunosuppressive therapy of patients with severe aplastic anemia. Ann Hematol. 2015;94(7):1105-1110

21. Yoshida N, Yagasaki H, Hama A, et al. Predicting response to immunosuppressive therapy in childhood aplastic anemia. Haematologica. 2011;96(5):771-774

22. Boddu P, Garcia-Manero G, Ravandi F, et al. Clinical outcomes in adult patients with aplastic anemia: a single institution experience. Am J Hematol. 2017:92(12):1295-1302.

23. Yoshizato T, Dumitriu B, Hosokawa K, et al Somatic mutations and clonal hematopoiesis in aplastic anemia. N Engl J Med. 2015;373(1):35-47.

24. Sugimori C, Chuhjo T, Feng X, et al. Minor population of CD55-CD59- blood cells predicts response to immunosuppressive therapy and prognosis in patients with aplastic anemia. Blood. 2006;107(4):1308-1314.

25. Kulagin A, Lisukov I, Ivanova $M$, et al Prognostic value of paroxysmal nocturnal haemoglobinuria clone presence in aplastic anaemia patients treated with combined immunosuppression: results of two-centre prospective study. Br J Haematol. 2014;164 (4):546-554

26. Sakaguchi H, Nishio N, Hama A, et al. Peripheral blood lymphocyte telomere length as a predictor of response to immunosuppressive therapy in childhood aplastic anemia. Haematologica. 2014:99(8): 1312-1316.

27. Kordasti S, Costantini B, Seidl T, et al. Deep phenotyping of Tregs identifies an immune signature for idiopathic aplastic anemia and predicts response to treatment. Blood. 2016;128(9):1193-1205

28. Zaimoku Y, Patel BA, Kajigaya S, et al Deficit of CD19+ CD24hi CD38hi regulatory $\mathrm{B}$ cells in severe aplastic anaemia. Br J Haematol. 2020;190(4):610-617.

29. Rosenfeld S, Follmann D, Nunez O, Young NS. Antithymocyte globulin and cyclosporine for severe aplastic anemia: association between hematologic response and long-term outcome. JAMA. 2003;289 (9):1130-1135.

30. Scheinberg P, Nunez O, Wu C, Young NS. Treatment of severe aplastic anaemia with combined immunosuppression: anti-thymocyte globulin, ciclosporin and mycophenolate mofetil. Br J Haematol. 2006;133(6):606611.

31. Scheinberg P, Wu CO, Nunez O, Boss C, Sloand EM, Young NS. Treatment of severe aplastic anemia with a combination of horse antithymocyte globulin and cyclosporine, with or without sirolimus: a prospective randomized study. Haematologica. 2009;94(3):348-354

32. Scheinberg $P$, Nunez $O$, Weinstein $B$,
Biancotto A, Wu CO, Young NS. Horse versus rabbit antithymocyte globulin in acquired aplastic anemia. N Engl J Med. 2011;365(5):430-438.

33. Feng X, Scheinberg $\mathrm{P}$, Wu CO, et al Cytokine signature profiles in acquired aplastic anemia and myelodysplastic syndromes. Haematologica. 2011;96(4):602606.

34. Kanda Y. Investigation of the freely available easy-to-use software 'EZR' for medical statistics. Bone Marrow Transplant. 2013;48(3): 452-458.

35. Bacigalupo A, Brand R, Oneto R, et al Treatment of acquired severe aplastic anemia: bone marrow transplantation compared with immunosuppressive therapythe European Group for Blood and Marrow Transplantation experience. Semin Hematol. 2000;37(1):69-80.

36. Yoshida N, Kobayashi R, Yabe H, et al. Firstline treatment for severe aplastic anemia in children: bone marrow transplantation from a matched family donor versus immunosuppressive therapy. Haematologica. 2014;99 (12):1784-1791.

37. Schrezenmeier $H$, Griesshammer $M$ Hornkohl A, et al. Thrombopoietin serum levels in patients with aplastic anaemia: correlation with platelet count and persisten elevation in remission. Br J Haematol. 1998;100(3):571-576.

38. Zhao X, Feng X, Wu Z, et al. Persistent elevation of plasma thrombopoietin levels after treatment in severe aplastic anemia. Exp Hematol. 2018;58:39-43.

39. Dunn DE, Tanawattanacharoen P, Boccuni P et al. Paroxysmal nocturnal hemoglobinuria cells in patients with bone marrow failure syndromes. Ann Intern Med. 1999;131(6): 401-408.

40. Katagiri T, Sato-Otsubo A, Kashiwase K, et al. Frequent loss of HLA alleles associated with copy number-neutral $6 \mathrm{pLOH}$ in acquired aplastic anemia. Blood. 2011:118 (25):6601-6609.

41. Zaimoku Y, Takamatsu H, Hosomichi K, et al. Identification of an HLA class I allele closely involved in the autoantigen presentation in acquired aplastic anemia. Blood. 2017;129(21):2908-2916.

42. Babushok DV, Duke JL, Xie HM, et al Somatic HLA mutations expose the role of class I-mediated autoimmunity in aplastic anemia and its clonal complications. Blood Adv. 2017;1(22):1900-1910.

43. Katagiri T, Kawamoto H, Nakakuki T, et al. Individual hematopoietic stem cells in human bone marrow of patients with aplastic anemia or myelodysplastic syndrome stably give rise to limited cell lineages. Stem Cells. 2013;31(3):536-546.

44. Maruyama H, Katagiri T, Kashiwase K, et al. Clinical significance and origin of leukocytes that lack HLA-A allele expression in patients with acquired aplastic anemia. Exp Hematol. 2016;44(10):931-939.

45. Lee-Six H, Obro NF, Shepherd MS, et al. Population dynamics of normal human blood inferred from somatic mutations. Nature. 2018;561(7724):473-478.

46. Admiraal R, van Kesteren C, Jol-van der Zijde CM, et al. Association between antithymocyte globulin exposure and CD4+ immune reconstitution in paediatric haemopoietic cell transplantation: a multicentre, retrospective pharmacodynamic cohort analysis. Lancet Haematol. 2015;2(5): e194-203

47. Admiraal R, Nierkens S, de Witte MA, et al Association between anti-thymocyte globu- 
lin exposure and survival outcomes in adult unrelated haemopoietic cell transplantation: a multicentre, retrospective, pharmacodynamic cohort analysis. Lancet Haematol. 2017;4(4):e183-e191.

48. Soiffer RJ, Kim HT, McGuirk J, et al. Prospective, randomized, double-blind, phase III clinical trial of anti-T-lymphocyte globulin to assess impact on chronic graftversus-host disease-free survival in patients undergoing HLA-matched unrelated myeloablative hematopoietic cell transplantation. J Clin Oncol. 2017;35(36):4003 4011.

49. Kennedy VE, Chen H, Savani BN, et al. Optimizing antithymocyte globulin dosing for unrelated donor allogeneic hematopoietic cell transplantation based on recipient absolute lymphocyte count. Biol Blood Marrow Transplant. 2018;24(1):150-155.
50. Shearer WT, Rosenblatt HM, Gelman RS, et al. Lymphocyte subsets in healthy children from birth through 18 years of age: the Pediatric AIDS Clinical Trials Group P1009 study. J Allergy Clin Immunol. 2003;112(5) 973-980.

51. McCarron M, Osborne Y, Story CI, Dempsey JL, Turner DR, Morley AA. Effect of age on lymphocyte proliferation. Mech Ageing Dev. 1987;41(3):211-218. 